primary outcome variable, consideration of PA, defined as clearly-documented consideration of PA in attending or trainee note OR skeletal survey ordered OR child-abuse team consult ordered. Co-variates examined as well.

Results Characteristics of 529 patients and physicians are displayed in Table 1. For the entire cohort, consideration of $\mathrm{PA}$ occurred in 346 (65\%), whereas consideration in infants $<6$ months of age occurred in 194 (78\%). EMR clearlydocumented consideration of PA occurred in 288 (54\%). Characteristics associated with greater odds for consideration of PA after covariate adjustment (OR and 95\% CI) included younger patient age (IQR of 12.1 to 38.7 weeks: 0.53 [0.33, $0.84]$ ), no history provided for injury $(9.41[3.88,22.82])$, softtissue injury $(4.95,[1.57,15.54])$, and male attending (1.84, $[1.12,3.01])$.

Conclusion PED physicians frequently do not consider PA in infants with fractures. Characteristics associated with consideration for PA include patient age, no history provided to explain the injury, soft-tissue injury, and male attending gender.

\section{Paediatric Intensive Care}

\section{0-096 NON-RESPIRATORY PELOD-2 SCORE IS A GOOD PREDICTOR OF MORTALITY IN CHILDREN WITH ACUTE RESPIRATORY FAILURE}

${ }^{1} S$ Leteurtre, ${ }^{2} \mathrm{~A}$ Duhamel, ${ }^{2} \mathrm{~V}$ Deken, ${ }^{1} \mathrm{C}$ Le Reun, ${ }^{3} \mathrm{~J}$ Lacroix, ${ }^{4} \mathrm{~F}$ Leclerc, ${ }^{1} \mathrm{Gfrup} .{ }^{7} \mathrm{PICU}$, CHRU de Lille, LILLE Cedex, France; ' ${ }^{2}$ Department of Biostatisitics, CHRU de Lille, LILLE Cedex, France; ${ }^{3}$ PICU, Sainte Justine Hospital, Montréal, Canada; ${ }^{4}$ Paris, GFRUP: Groupe Francophone de Reanimation Et Urgences Pediatriques, France

\subsection{6/archdischild-2014-307384.163}

Background and aim Multiple organ dysfunction, not respiratory failure, is the major cause of death in children with ALI or ARDS. This study was undertaken to estimate the predictive value of death of the non-respiratory Paediatric Logistic Organ Dysfunction (PELOD)-2 (NRespPELOD-2) in children with acute respiratory failure (ARF).

Methods Analysis of the database of the recently published PELOD-2. All consecutive children (excluding neonates) admitted to 9 PICU in France and Belgium (June 2006-October 2007) and having ARF. We prospectively collected data on variables considered for the PELOD-2 score during PICU stay: days 1, 2, 5, 8, 12, 16 and 18, plus PICU discharge. For each variable of the PELOD-2 score, the most abnormal value observed during time points was collected. Outcome was vital status at PICU discharge. We used AUCs to estimate the discrimination and Hosmer-Lemeshow goodness-of-fit tests to estimate calibration of the PELOD-2 and the NRespPELOD-2 scores, with correction for the optimism bias using a bootstrap resampling method.

Results We included 1572 patients (median age: 20.6 months; mortality: 9.5\%). Discrimination of the PELOD-2 and the NRespPELOD-2 was excellent (AUC $=0.93$ and 0.92, respectively) and calibration was good ( $\mathrm{p}=0.45$ and 0.27 , respectively). The four NResp organ dysfunctions were closely related to the risk of mortality $(\mathrm{p}<0.001)$.

Conclusions Our study demonstrates that the NRespPELOD-2 score of the entire PICU stay is highly predictive of death in children with ARF of whom $94.3 \%$ were invasively ventilated. It could represent the non-respiratory organ failure definition tool claimed by the international experts on paediatric ARDS.

\section{0-097 CLINICAL SCORE TO PREDICT OUTCOME IN CONGENITAL DIAPHRAGMATIC HERNIA; RESULTS OBTAINED FROM AN INTERNATIONAL MULTICENTER STUDY}

${ }^{1} \mathrm{KG}$ Snoek, ${ }^{2} \mathrm{C}$ Capolupo, ${ }^{2} \mathrm{~F}$ Morini, ${ }^{1} \mathrm{RMH}$ Wijnen, ${ }^{3} \mathrm{~K} \mathrm{KM}$ Reiss, ${ }^{2} \mathrm{P}$ Bagolan, ${ }^{1} \mathrm{H}$ IJsselstijn, ${ }^{1} \mathrm{D}$ Tibboel. 'Department of Paediatric Surgery and Intensive Care, Erasmus MC Sophia Children's Hospital, Rotterdam, Netherlands; '2Department of Medical and Surgical Neonatology, Bambino Gesu Children's Hospital, Rome, Italy; ${ }^{3}$ Department of Neonatology, Erasmus MC Sophia Children's Hospital, Rotterdam, Netherlands

\subsection{6/archdischild-2014-307384.164}

Background In congenital diaphragmatic hernia $(\mathrm{CDH})$ reliable postnatal predictors of outcome are scarce. Score for Neonatal Acute Physiology-II (SNAP-II) (Richardson, 2001) has been validated to predict mortality in newborns. We hypothesised that higher SNAP-II scores are found in non-survivors, those who need extracorporeal membrane oxygenation (ECMO), and those who develop chronic lung disease (CLD), defined as oxygen dependency at day 28.

Methods In a prospective, randomised controlled multicenter trial initiated by the Euroconsortium (VICI-trial, NTR 1310), neonates born between November 2008 and December 2013, were randomised for initial ventilation strategy (either high-frequency oscillation or conventional mechanical ventilation). Prenatal, perinatal and postnatal data including SNAP-II scores were collected. Mann-Whitney U test was used for analyses. Data were presented as $\mathrm{n}(\%)$ and median (IQR).

Results Of the 173 included patients, 46 patients (26.6\%) died, $39(22.5 \%)$ underwent ECMO, and 38 patients (29.9\%) of the survivors developed CLD. Overall, the SNAP-II score was 22.5 (10 to 37). In non-survivors SNAPP-II score was 40.0 (32.0 to 54.5 ) and 16.5 (7.8 to 26.5 ) in survivors ( $p<0.001)$. It was 35.0 (30.0 to 46.0) in ECMO treated patients and 19.0 (9.0 to $32.0)$ in patients without ECMO need $(\mathrm{p}<0.001)$. Survivors with CLD had SNAP-II score of 25.5 (21.0 to 35.5) and those without CLD 13.0 (5.0 to21.0) $(\mathrm{p}<0.001)$.

Conclusions In this prospective multicenter study, we showed that in CDH patients SNAP-II scores may not only be useful to predict mortality but also to identify patients who need ECMO or are at risk for development of CLD.

\section{Pharmacology I}

\section{0-098 POTENTIALLY HARMFUL EXCIPIENTS IN MEDICINES PRESCRIBED IN NEONATAL INTENSIVE CARE UNITS (NICU) - PRODUCT SUBSTITUTION AS A WAY FORWARD}

${ }^{1} \mathrm{G}$ Nellis, ${ }^{2}$ Lutsar, ${ }^{1} \mathrm{H}$ Varendi, ${ }^{3} \mathrm{~J}$ Lass, ${ }^{4} \mathrm{MA}$ Turner, ${ }^{5} \mathrm{~T}$ Metsvaht. ${ }^{1}$ Neonatal Unit, Children's Clinic of Tartu University Hospital, Tartu, Estonia; ${ }^{2}$ Department of Microbiology, Tartu University, Tartu, Estonia; ${ }^{3}$ Pharmacy Department, Tartu University Hospital, Tartu, Estonia; ${ }^{4}$ Neonatal Unit, Liverpool Women's Hospital, Liverpool, UK; ${ }^{5}$ Paediatric Intensive Care Unit, Tartu University, Hospital, Tartu, Estonia

\subsection{6/archdischild-2014-307384.165}

Background Excipients are essential for many medicines. Some have been associated with significant consequences in neonates. We aimed to identify substitution possibilities among medicines used in European neonates in order to minimise the administration of potentially harmful excipients of interest (EOI).

Methods A 3-day survey recording all medicines prescribed to neonates was performed in European NICUs. Based on existing toxicity data in neonates the EOI included parabens, polysorbate 80, propylene glycol, benzoates, saccharin sodium, sorbitol, 